SUPPORTING INFORMATION FOR PUBLICATION

\title{
Structural Identification of Monounsaturated Branched Chain Fatty Acid Methyl Esters by Combination of Electron Ionization and Covalent Adduct Chemical Ionization Tandem Mass Spectrometry
}

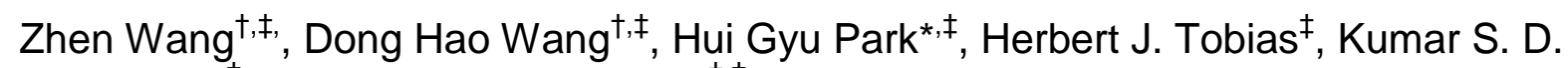

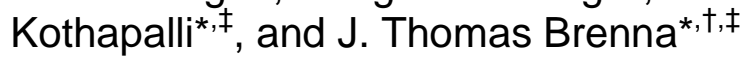

${ }^{\dagger}$ Department of Food Science, Cornell University, Ithaca, New York 14853, USA

${ }^{\ddagger}$ Dell Pediatric Research Institute, Departments of Pediatrics, of Chemistry, and of Nutrition, The University of Texas at Austin, 1400 Barbara Jordan Blvd, Austin, TX 78723, USA

The Supporting Information contains the following:

Table S1. Precursor ions and collision energy applied for MS/MS analysis of tested FAME.

Figure S1. El-MS/MS spectra of isomeric 17:0 and isomeric 19:0 FAME. (A) n-17:0; (B) iso17:0; (C) anteiso-17:0; (D) n-19:0; (E) iso-19:0; (F) anteiso-19:0.

Figure S2. $\mathrm{CI}-\mathrm{MS} / \mathrm{MS}$ spectra at collision energies $3 \mathrm{~V}$ and $18 \mathrm{~V}$ of isomeric 17:0 FAME fragmented from $[\mathrm{MH}]^{+}$at $\mathrm{m} / \mathrm{z} 2285$. (A) $n-17: 0$ (3V); (B) iso-17:0 (3V); (C) anteiso-17:0 (3V); (D) n-17:0 (18V); (E) iso-17:0 (18V); (F) anteiso-17:0 (18V).

Figure S3. CI-MS/MS spectra at collision energies $3 \mathrm{~V}, 6 \mathrm{~V}$ and $18 \mathrm{~V}$ of isomeric $17: 1 \mathrm{FAME}$ fragmented from [MH] ${ }^{+}$at $\mathrm{m} / \mathrm{z}$ 283. (A) $n-6 Z-17: 1$ (3V); (B) iso-6Z-17:1 (3V); (C) anteiso-6Z17:1 (3V); (D) n-6Z-17:1 (6V); (E) iso-6Z-17:1 (6V); (F) anteiso-6Z-17:1 (6V); (G) n-6Z-17:1 (18V); $(\mathrm{H})$ iso-6Z-17:1 (18V); (I) anteiso-6Z-17:1 (18V).

Figure S4. $m / z 141$ is present in El-MS/MS spectra of MUFAME and monocyclopropane FAME (A) Elaidic acid 9E-18:1; (B) Oleic acid 9Z-18:1; (C) Cis-vaccenic acid 11Z-18:1; (D) 7,8-cyclopropane-17:0; (E) 9,10-cyclopropane-19:0.

*Corresponding authors

<tbrenna@utexas.edu>, v. 512-495-5249;

<kkothapalli@utexas.edu>, v. 512-495-5950;

<hi.park@austin.utexas.edu>, v.607-280-7234. 
31 Table S1. Precursor ions and collision energy applied for MS/MS analysis of tested FAME.

$\begin{array}{cccccc}\begin{array}{c}\text { FAME } \\ (n-, \text { iso- and } \\ \text { anteiso- })\end{array} & \begin{array}{c}\text { Molecular Mass } \\ (\mathrm{m} / \mathrm{z})\end{array} & \begin{array}{c}\text { Erecursor Ion } \\ {[\mathrm{M}]^{+}(\mathrm{m} / \mathrm{z})}\end{array} & \begin{array}{c}\text { Collision energy } \\ (\mathrm{V})\end{array} & \begin{array}{c}\text { Precursor Ion } \\ {[\mathrm{M}+54]^{+}(\mathrm{m} / \mathrm{z})}\end{array} & \begin{array}{c}\text { Collision energy } \\ (\mathrm{V})\end{array} \\ 15: 1 & 254 & 254 & 3 & 308 & 6 \\ 16: 1 & 268 & 268 & 3 & 322 & 6 \\ 17: 1 & 282 & 282 & 3 & 36 & 6 \\ 18: 1 & 296 & 296 & 3 & 350 & 6 \\ 19: 1 & 310 & 310 & 3 & 364 & 6 \\ 20: 1 & 324 & 324 & 3 & 378 & 6\end{array}$

32

33 
(A) El-MS/MS n-17:0

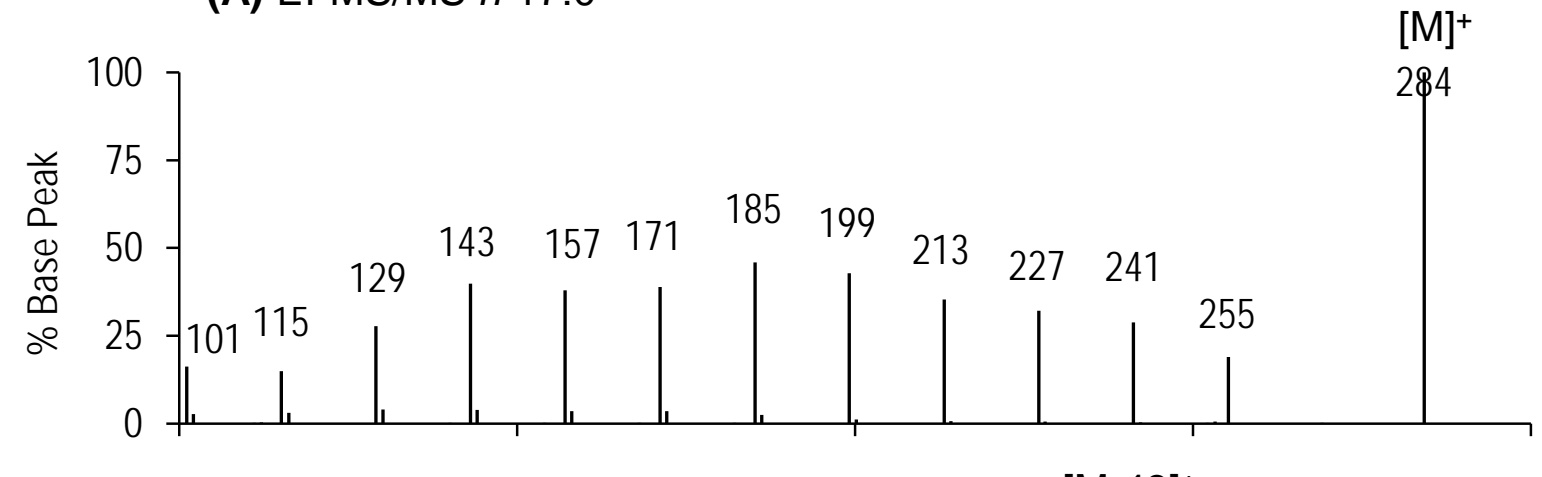

(B) El-MS/MS iso-17:0

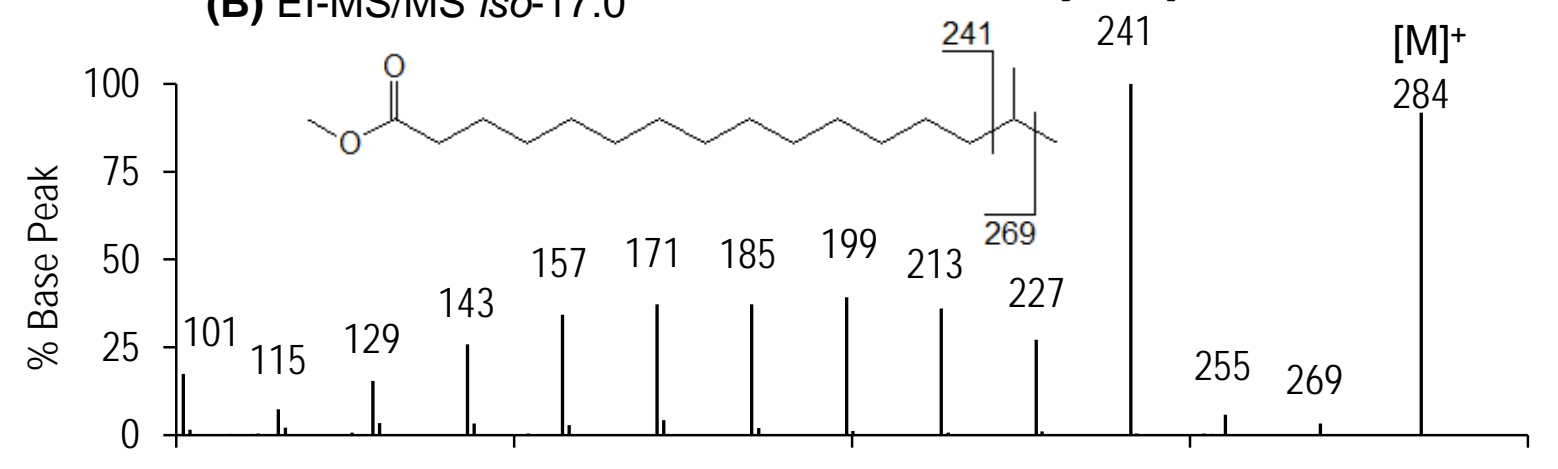

(C) El-MS/MS anteiso-17:0 [M-57 $]^{+}$

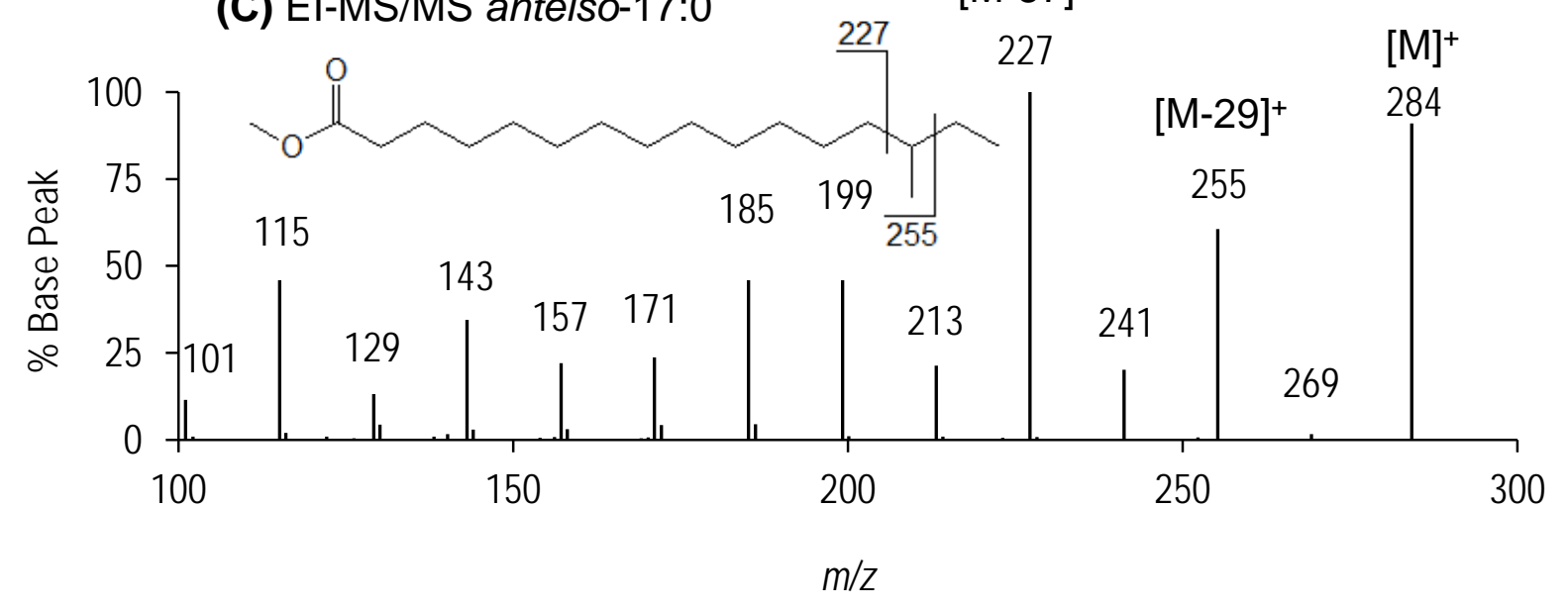

34 
(D) El-MS/MS n-19:0

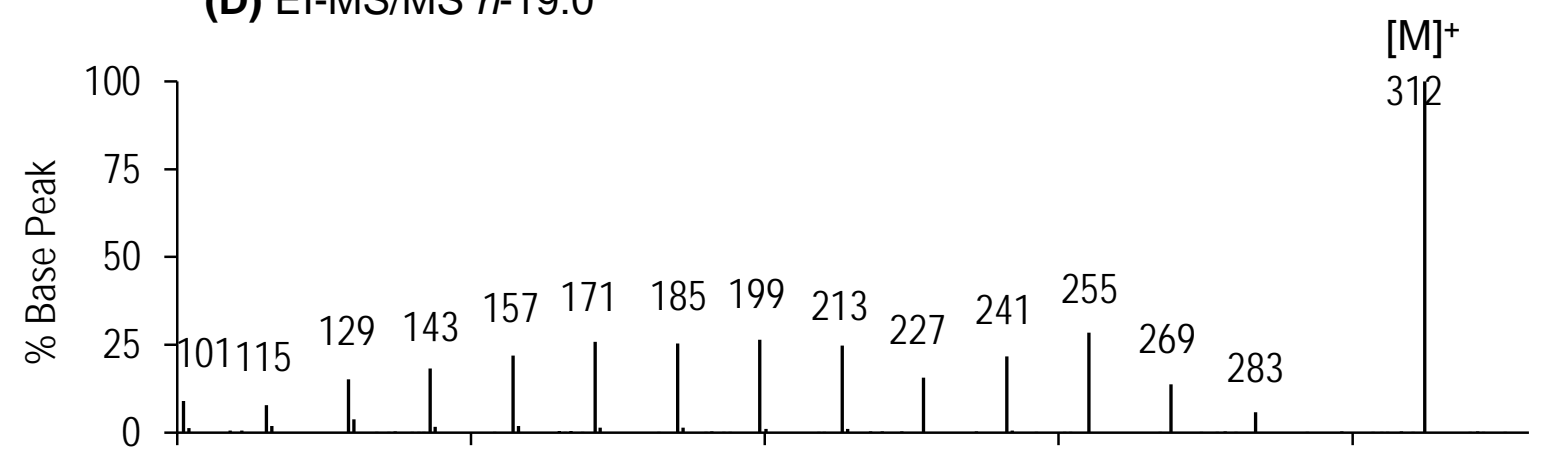

(E) El-MS/MS iso-19:0

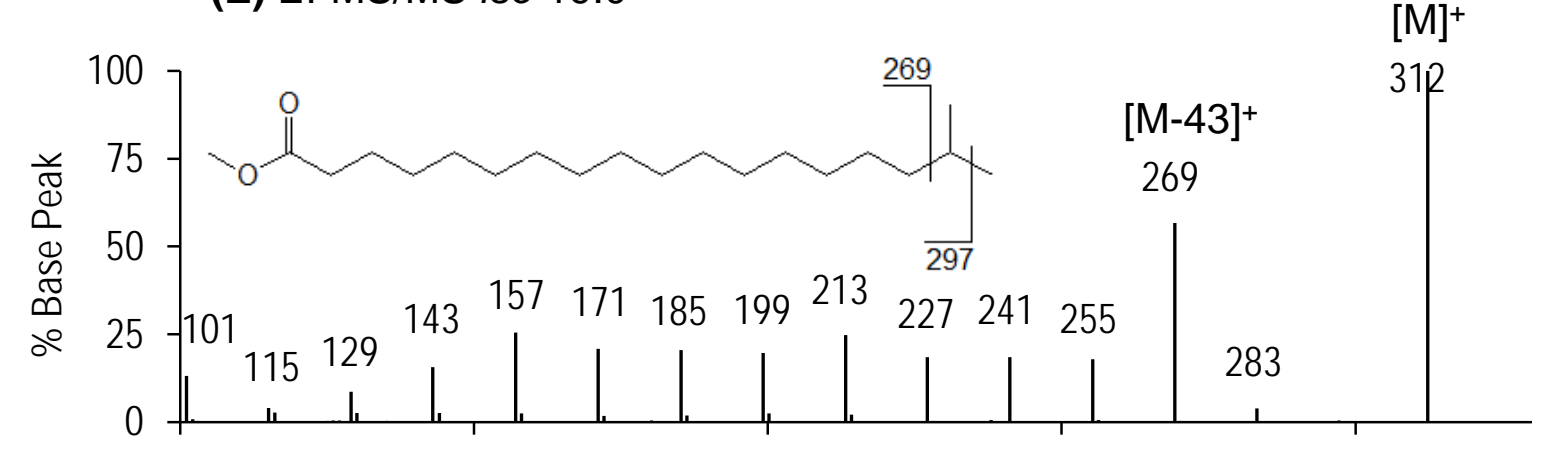

(F) El-MS/MS anteiso-19:0 [M-57]

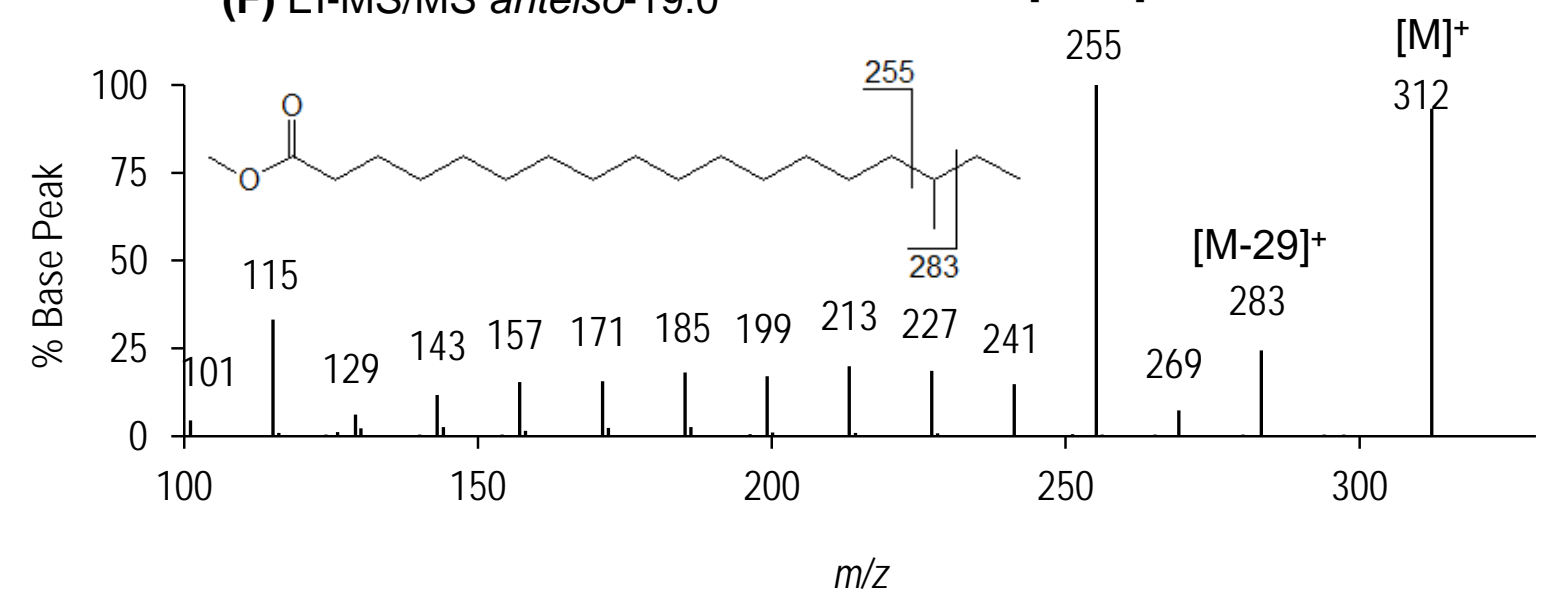

35

36

37

38

39

40 
(A) $\mathrm{Cl}-\mathrm{MS} / \mathrm{MS} n-17: 0$ at $3 \mathrm{~V}$

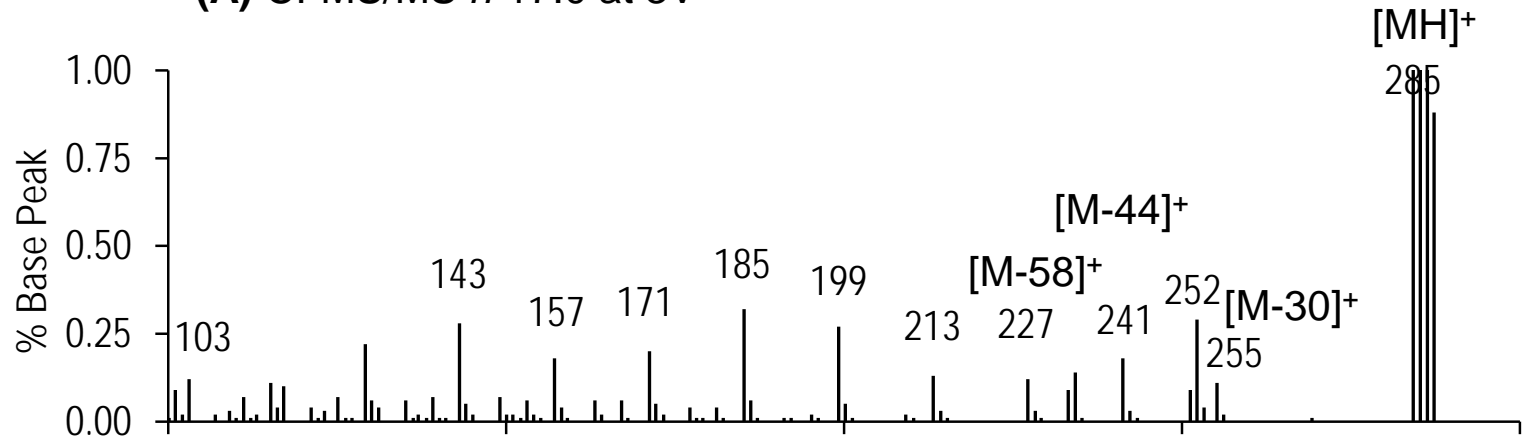

(B) Cl-MS/MS iso-17:0 at 3V

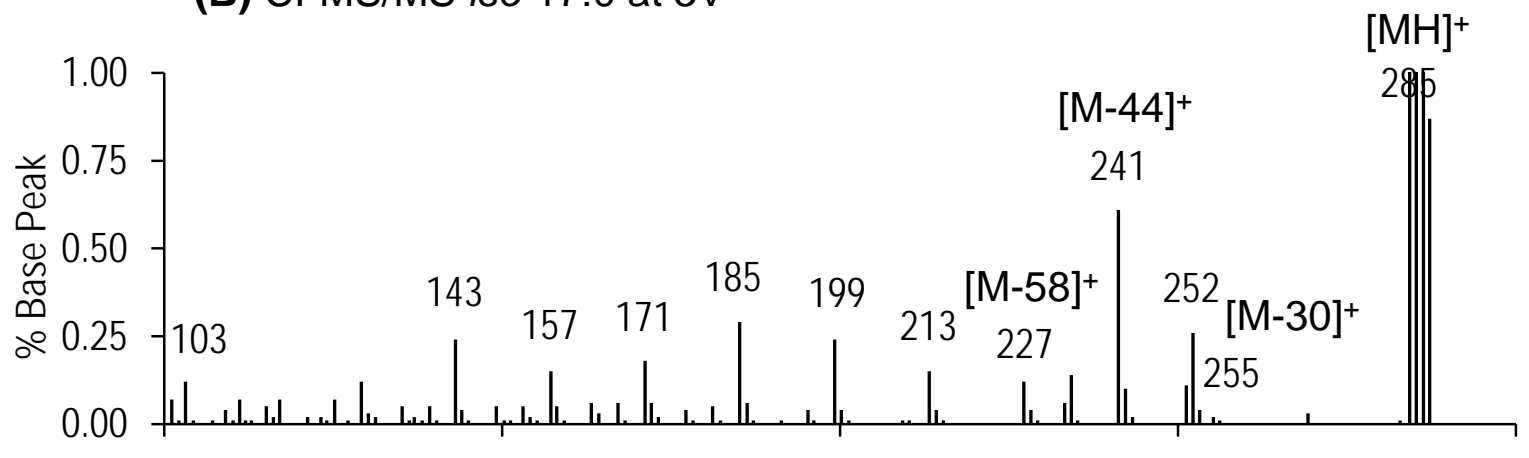

(C) CI-MS/MS anteiso-17:0 at 3V

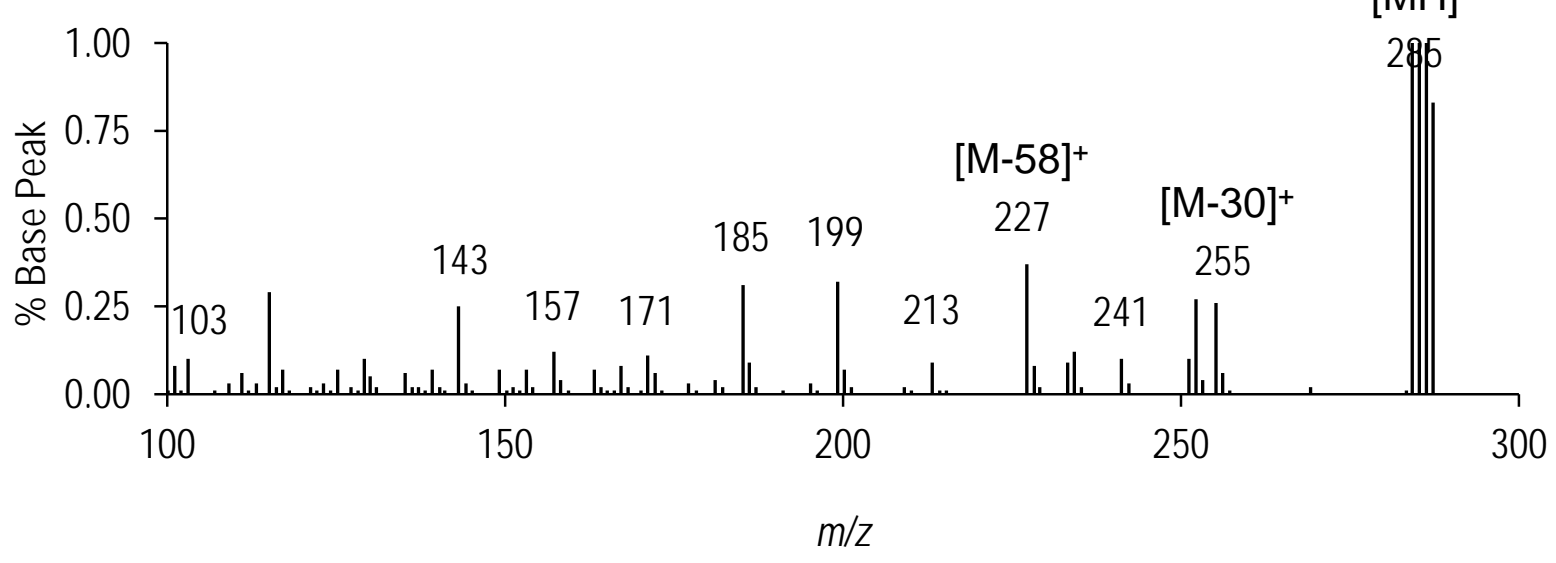


(D) $\mathrm{Cl}-\mathrm{MS} / \mathrm{MS} n-17: 0$ at $18 \mathrm{~V}$

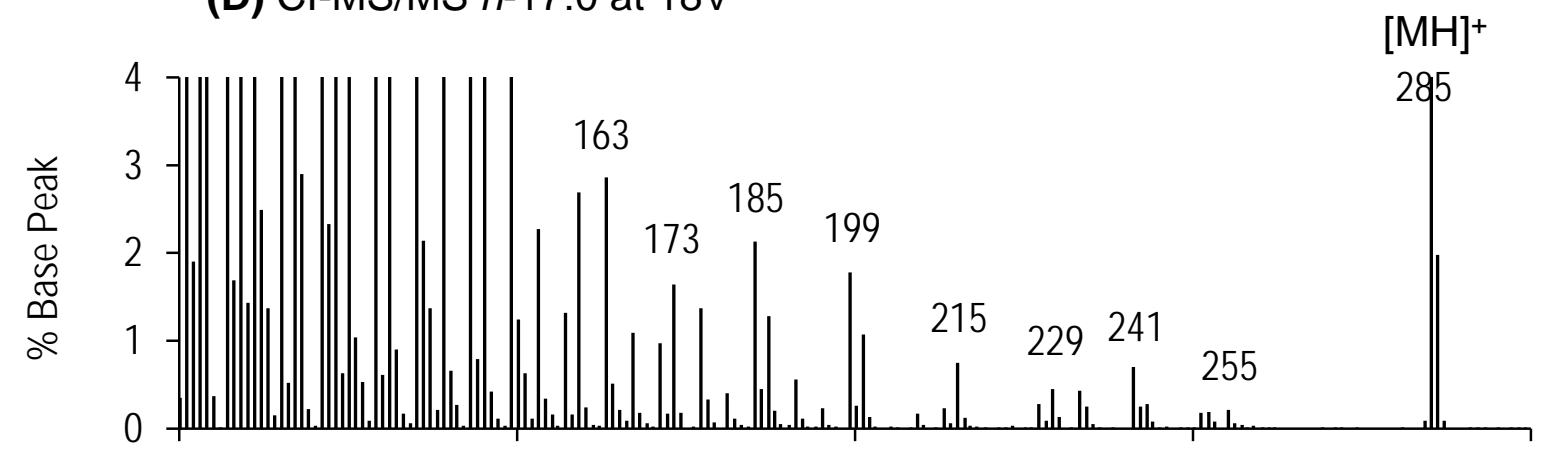

(E) Cl-MS/MS iso-17:0 at $18 \mathrm{~V}$

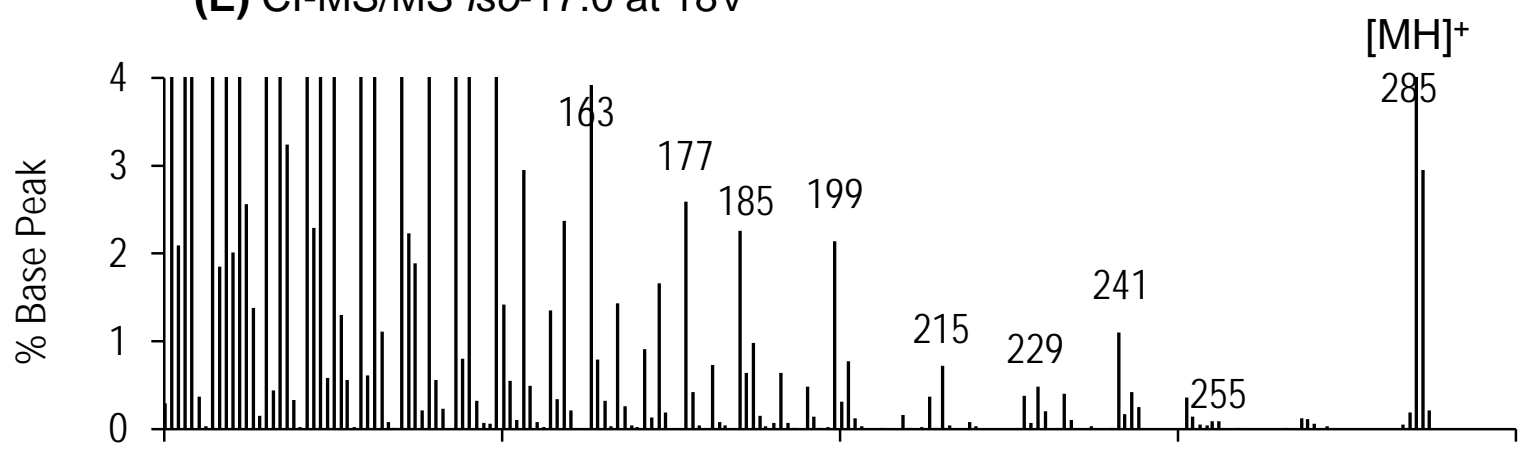

(F) Cl-MS/MS anteiso-17:0 at 18V

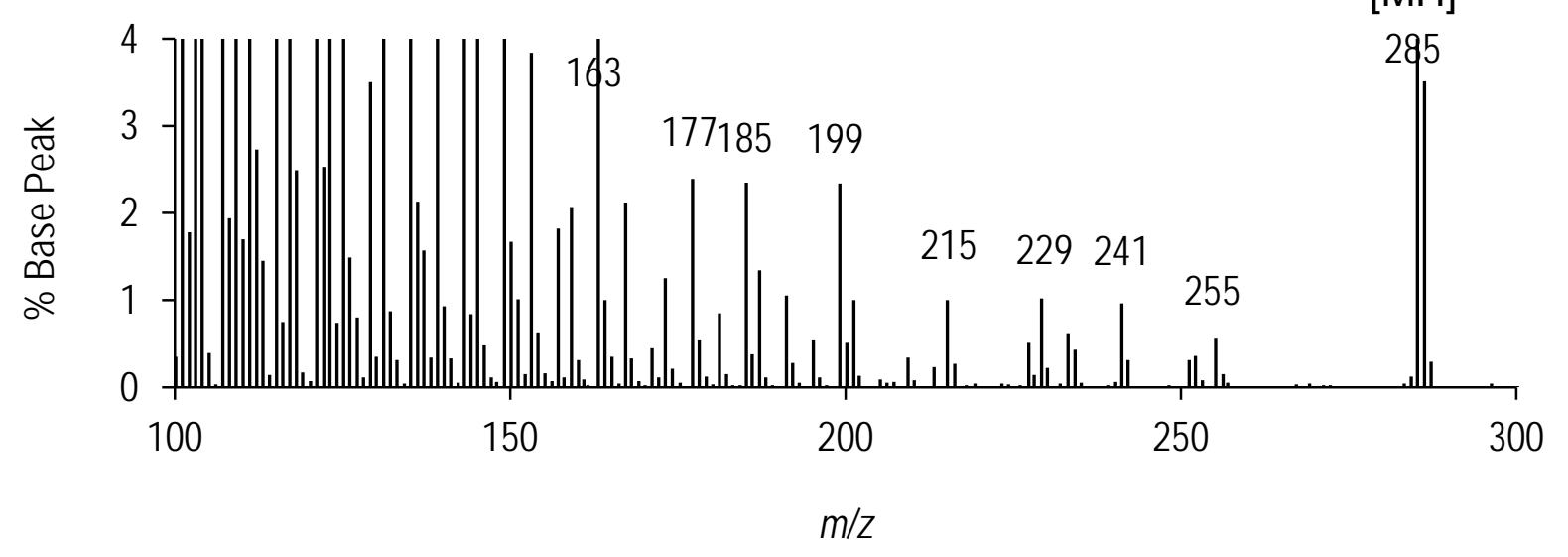

42

43

44

45

46

47

48

49

Figure S2. $\mathrm{Cl}-\mathrm{MS} / \mathrm{MS}$ spectra at collision energies $3 \mathrm{~V}$ and 18V of isomeric 17:0 FAME fragmented from $[\mathrm{MH}]^{+}$at $\mathrm{m} / \mathrm{z} 285$. (A) $n-17: 0$ (3V); (B) iso-17:0 (3V); (C) anteiso-17:0 (3V); (D) $n-17: 0$ (18V); (E) iso-17:0 (18V); (F) anteiso-17:0 (18V). Compared to collision energy at $6 \mathrm{~V}$ presented in Figure 9, $\mathrm{Cl}-\mathrm{MS} / \mathrm{MS}$ at $3 \mathrm{~V}$ yield similar but lower abundance of characteristic ions for assigning branched ends of isomeric $17: 0.18 \mathrm{~V}$ is too high to generate useful characteristic ions as $3 \mathrm{~V}$ and $6 \mathrm{~V}$. 


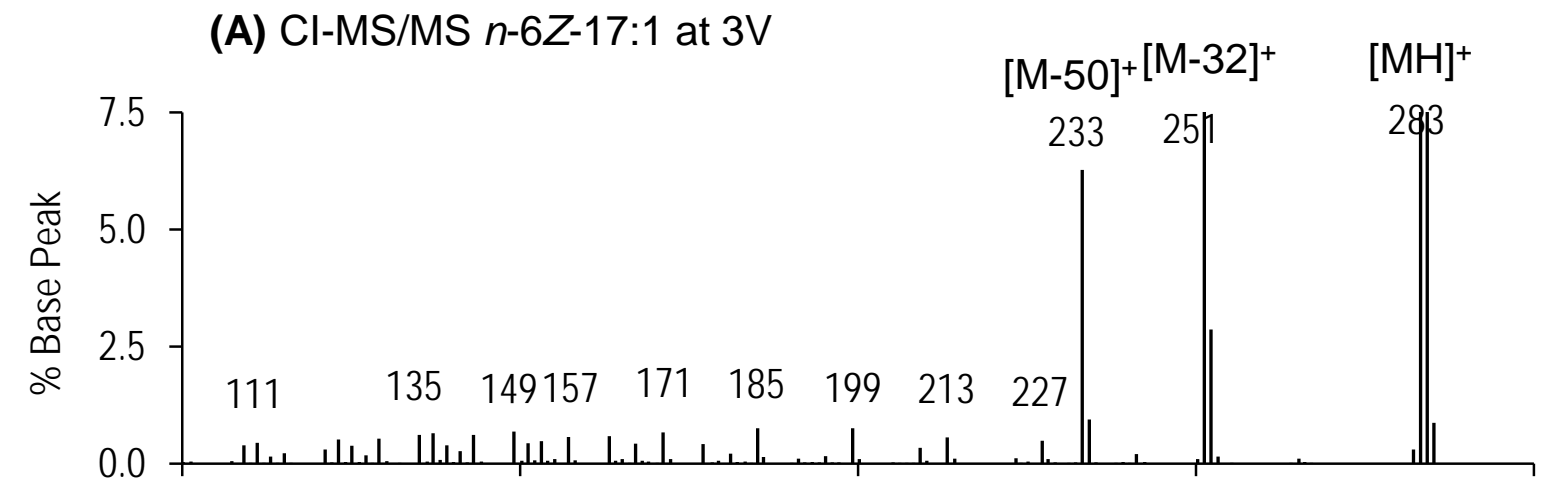

(B) $\mathrm{Cl}-\mathrm{MS} / \mathrm{MS}$ iso-6Z-17:1 at $3 \mathrm{~V}$

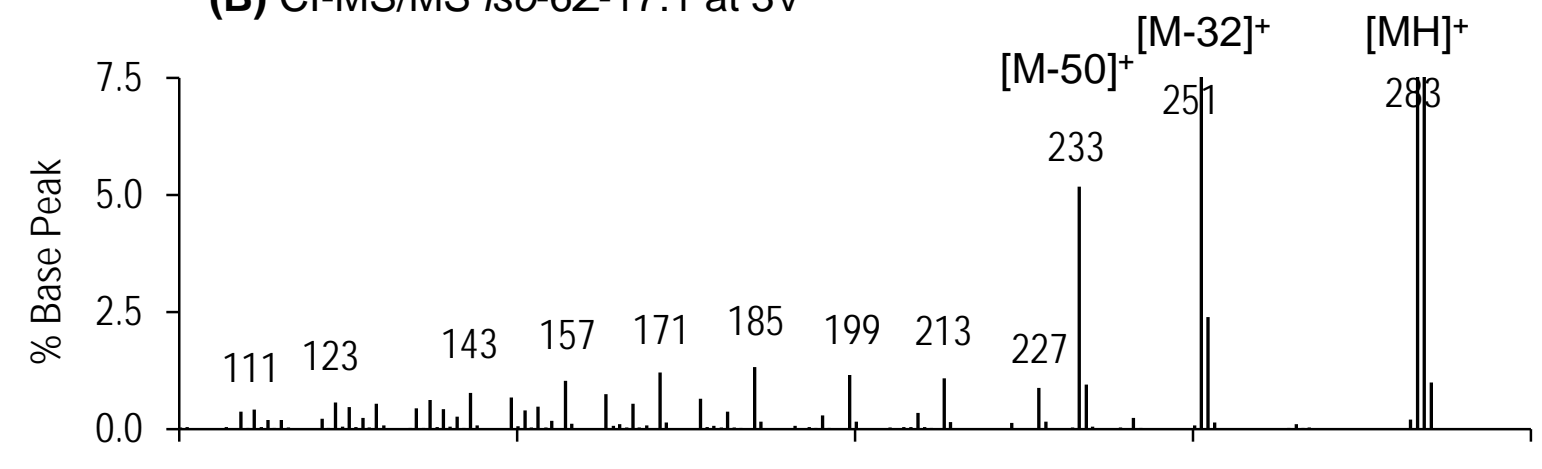

(C) Cl-MS/MS anteiso-6Z-17:1 at 3V

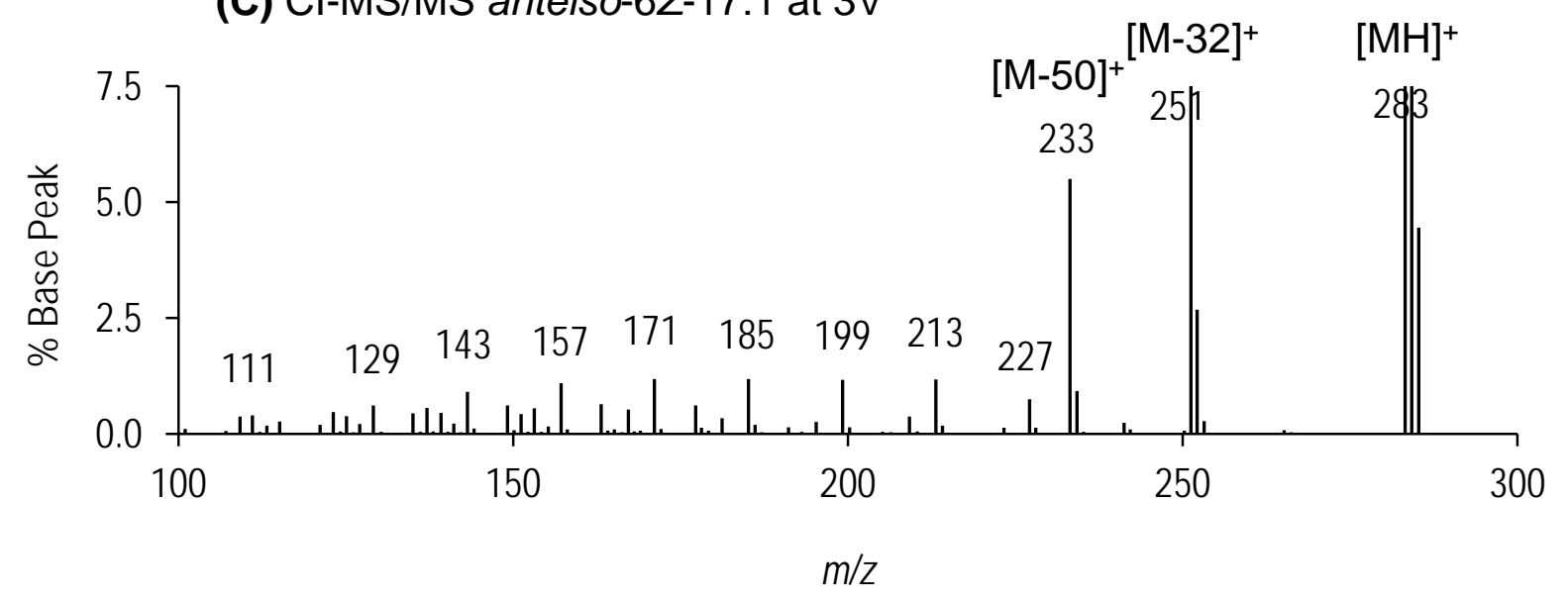

50 
(D) $\mathrm{Cl}-\mathrm{MS} / \mathrm{MS} n-6 Z-17: 1$ at $6 \mathrm{~V}$

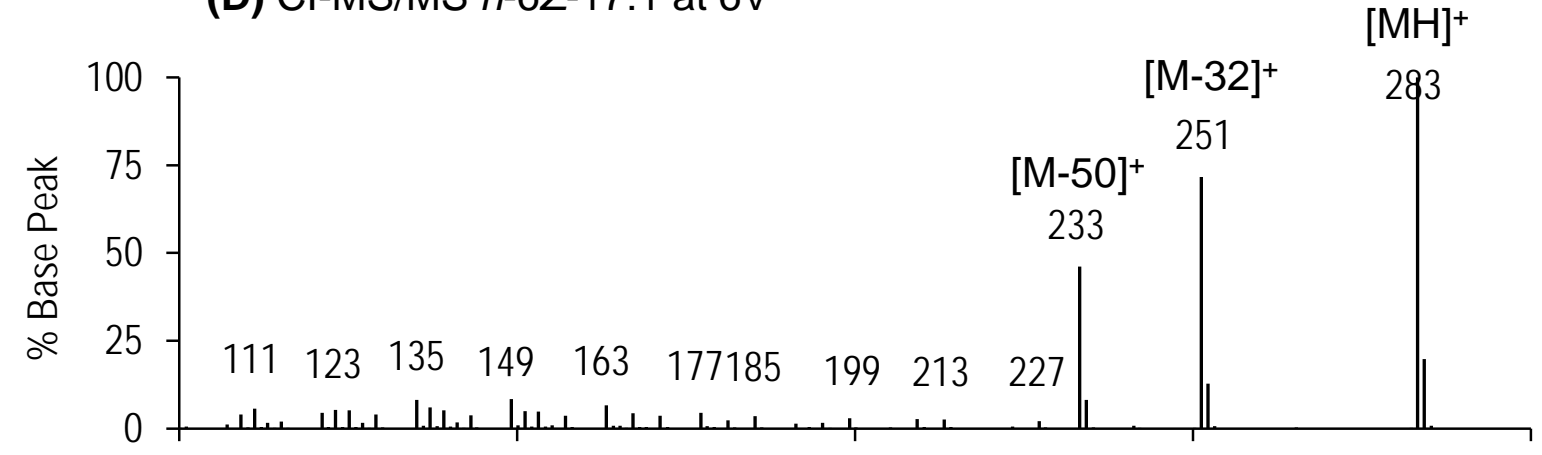

(E) $\mathrm{Cl}-\mathrm{MS} / \mathrm{MS}$ iso-6Z-17:1 at $6 \mathrm{~V}$

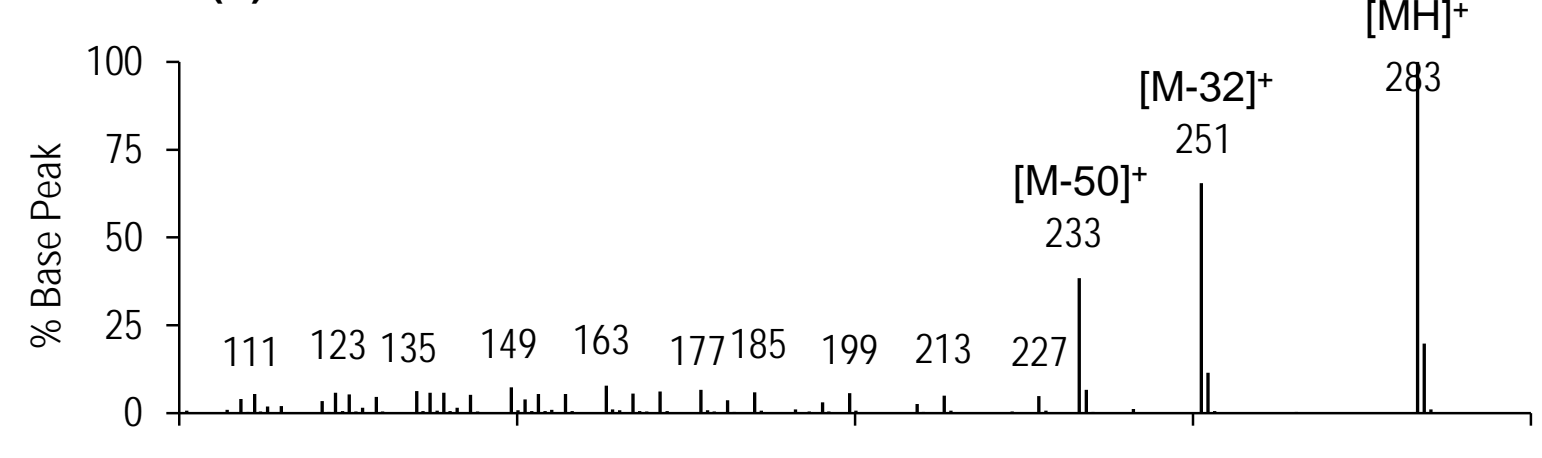

(F) $\mathrm{Cl}-\mathrm{MS} / \mathrm{MS}$ anteiso-6Z-17:1 at $6 \mathrm{~V}$

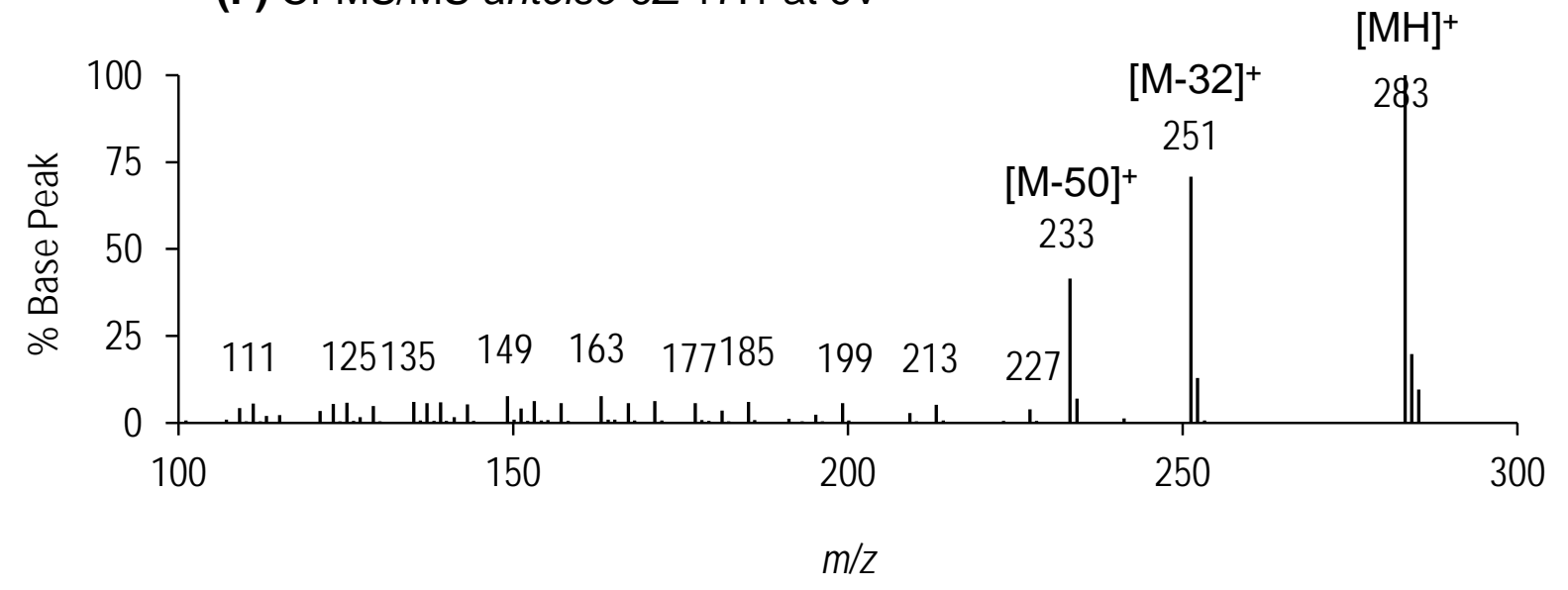


(G) Cl-MS/MS n-6Z-17:1 at $18 \mathrm{~V}$

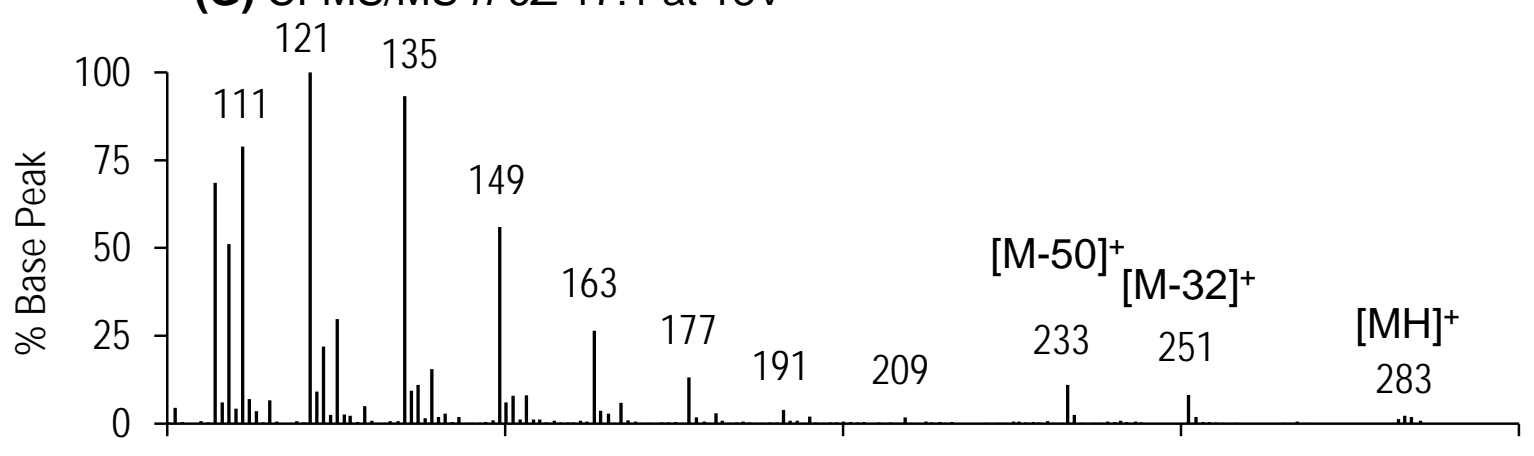

(H) Cl-MS/MS iso-6Z-17:1 at $18 \mathrm{~V}$

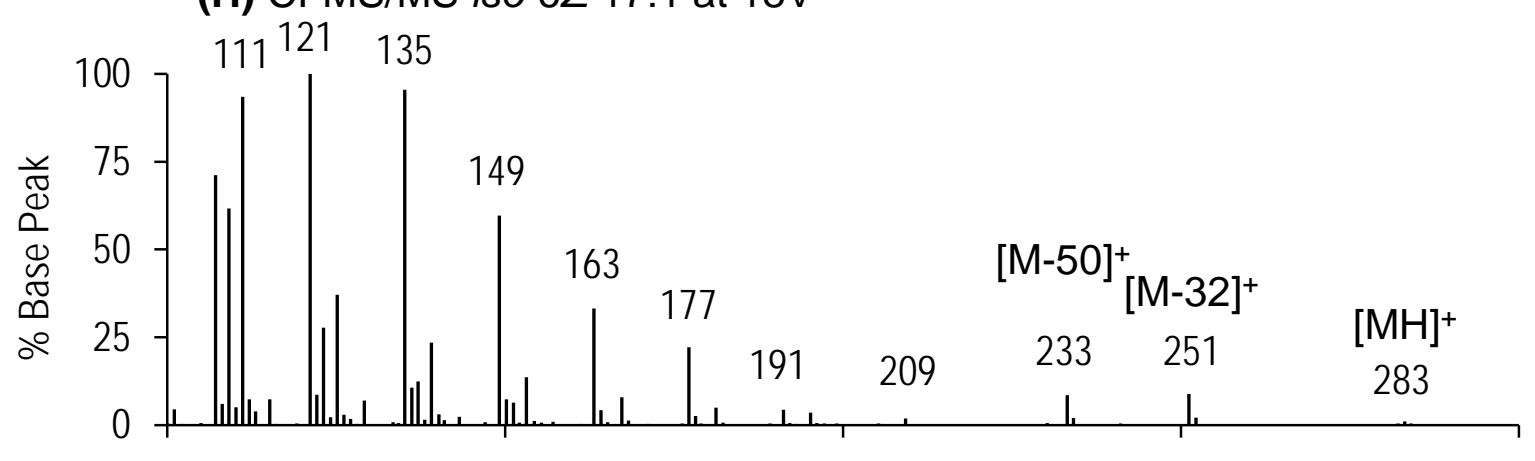

(I) Cl-MS/MS anteiso-6Z-17:1 at 18V

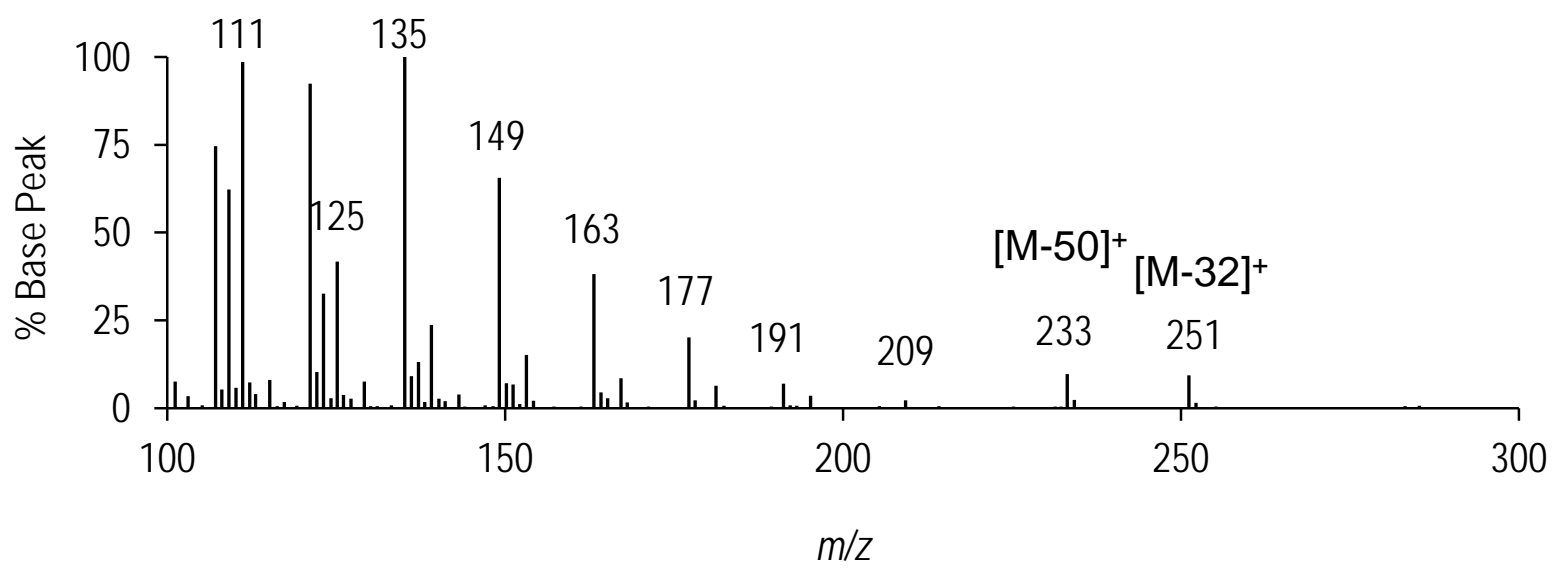

52

53

54

55

56

57

58

59

60
Figure S3. CI-MS/MS spectra at collision energies $3 \mathrm{~V}, 6 \mathrm{~V}$ and $18 \mathrm{~V}$ of isomeric $17: 1 \mathrm{FAME}$ fragmented from $[\mathrm{MH}]^{+}$at $\mathrm{m} / \mathrm{z}$ 283. (A) $n-6 Z-17: 1$ (3V); (B) iso-6Z-17:1 (3V); (C) anteiso-6Z17:1 (3V); (D) $n-6 Z-17: 1$ (6V); (E) iso-6Z-17:1 (6V); (F) anteiso-6Z-17:1 (6V); (G) $n-6 Z-17: 1$ (18V); (H) iso-6Z-17:1 (18V); (I) anteiso-6Z-17:1 (18V). Isomeric 17:1 share the most abundant ions [MH-32] ${ }^{+}$and $[\mathrm{MH}-50]^{+}$at $\mathrm{m} / \mathrm{z} 251$ and 233 when collision energy is $3 \mathrm{~V}$ or $6 \mathrm{~V}$. After careful inspection, no unique ions are found to distinguish isomeric $17: 1$ at either $3 \mathrm{~V}, 6 \mathrm{~V}$ or $18 \mathrm{~V}$. 
(A) El-MS/MS Elaidic acid 9E-18:1

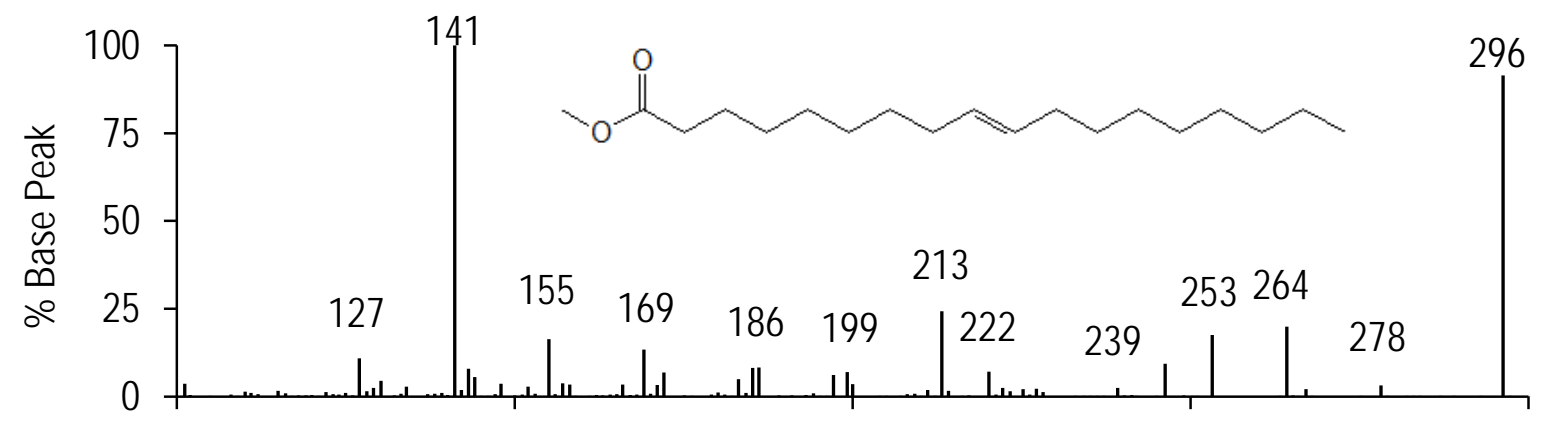

(B) EI-MS/MS Oleic acid 9Z-18:1

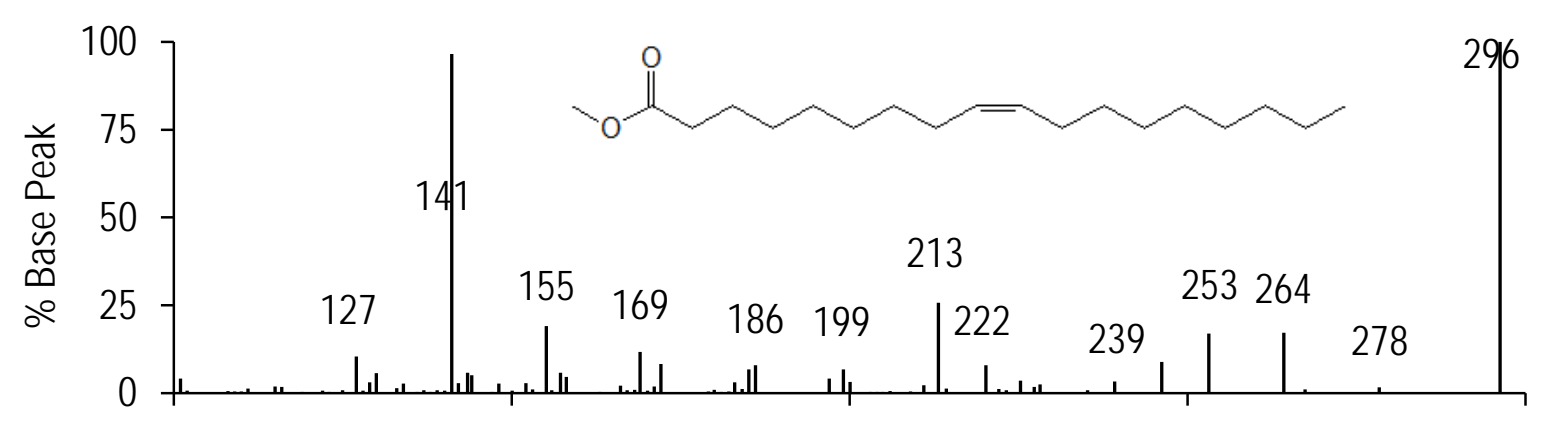

(C) El-MS/MS Cis-vaccenic acid 11Z-18:1

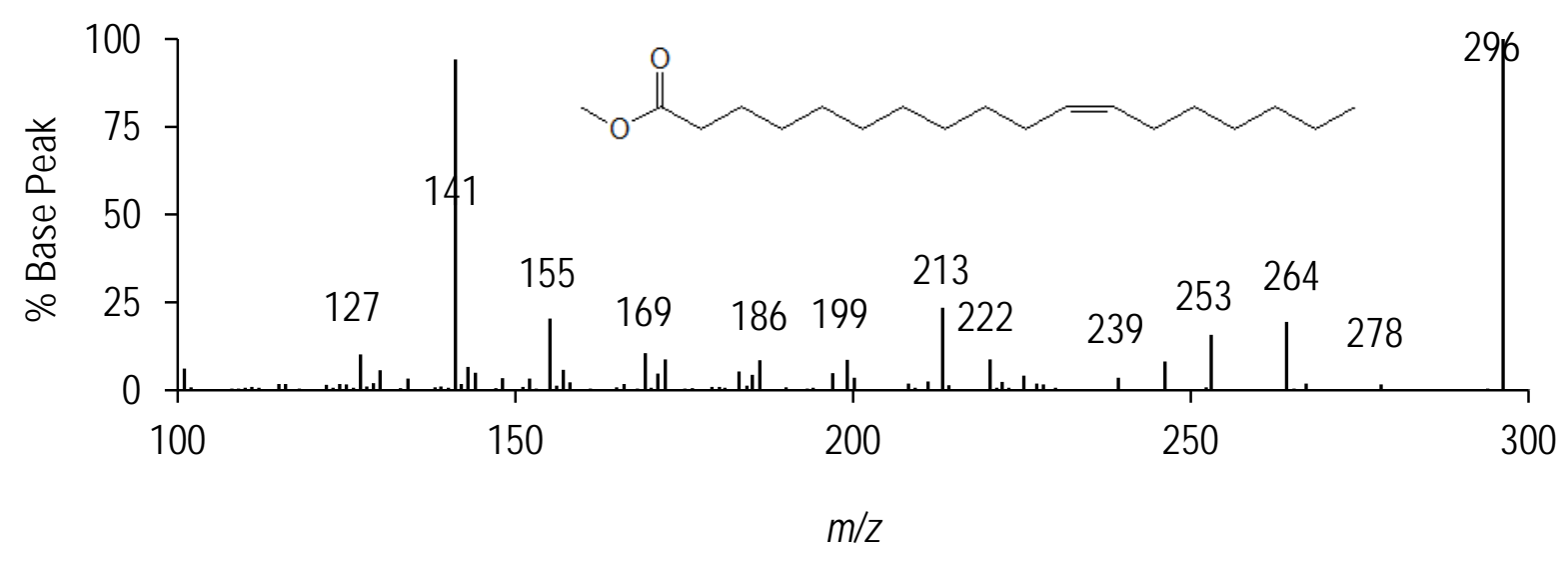


(D) El-MS/MS 7,8-cyclopropane-17:0

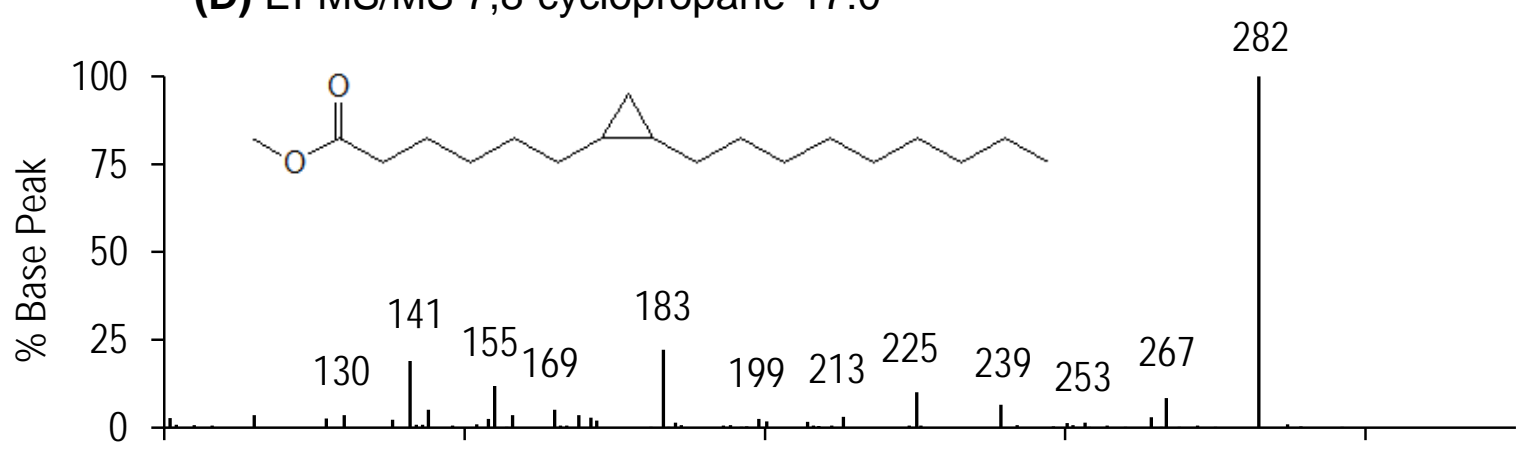

(E) EI-MS/MS 9,10-cyclopropane-19:0

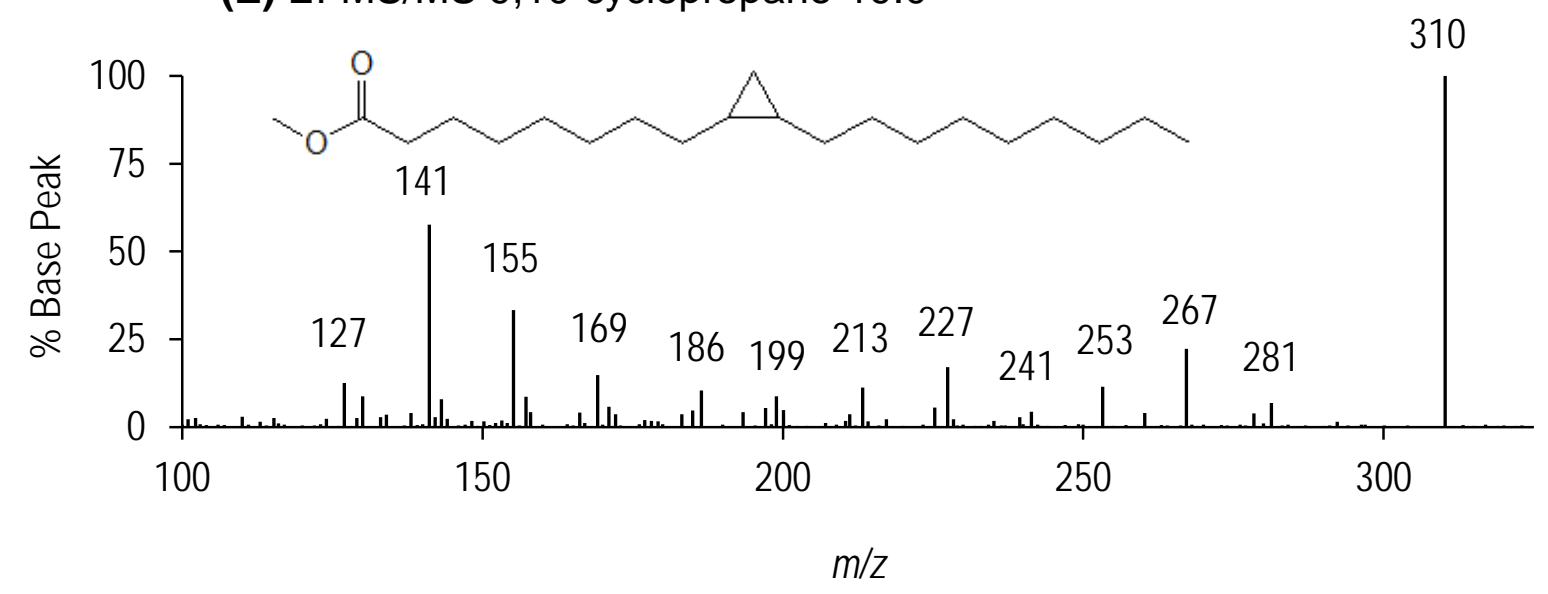

62

63

64

65

66
Figure S4. $m / z 141$ is present in El-MS/MS spectra of MUFAME and monocyclopropane FAME (A) Elaidic acid 9E-18:1; (B) Oleic acid 9Z-18:1; (C) Cis-vaccenic acid 11Z-18:1; (D) 7,8-cyclopropane-17:0; (E) 9,10-cyclopropane-19:0. 